\title{
MULTI-COMPARTMENT MODELS
}

\author{
ABDERRAHMAN IGGIDR \\ Laboratoire de Mathématiques et Applications \\ UMR CNRS 7122, University of Metz and INRIA Lorraine \\ Metz, France \\ Joseph Mbang \\ University of Yaoundé I \\ Cameroon \\ Gauthier Sallet \\ Laboratoire de Mathématiques et Applications \\ UMR CNRS 7122, University of Metz and INRIA Lorraine \\ Metz, France \\ JEAN-Jules TEWA \\ University of Yaoundé I \\ Cameroon
}

\begin{abstract}
We consider models with a general structure which, for example, encompasses the so-called DI, SP or DISP models with mass action incidence. We give a very simple formule for the basic reproduction ratio $\mathcal{R}_{0}$. If $\mathcal{R}_{0} \leq 1$ we prove that the disease free equilibrium is globally asymptotically stable on the nonnegative orthant. If $\mathcal{R}_{0}>1$, we prove the existence of a unique endemic equilibrium in the positive orthant and give an explicit formula. We prove the global asymptotic stability of the endemic equilibrium, when $\mathcal{R}_{0}>1$ for SP model.
\end{abstract}

1. Introduction. The objective of this paper is to provide a stability analysis for models with a general structure and mass action incidence. The structure can deal with various complex interactions between different classes of infectiousness. We do not consider difference in susceptibilities as it is the case for example in [10]. We also consider mass action incidence. Mass action incidence is plausible in number of cases ( see e.g [15]) or when variables considered are concentration (as number by unit area). Or as was pointed out in [21,38], if the number of contacts per person, in general, is a function of the population size. For certain diseases, such as influenza and measles, or in certain ranges of population sizes, it is appropriate to assume that the number of contacts is proportional to the population size. Then the rate of infection has a bilinear form.

Our structure can handle models with different classes of latency and infectiousness. Models with variation in infectiousness have been considered since a long time in the literature $[28,1,26,42,43]$. Many reasons can be invoked to consider such models. If the infectiousness changes over the infectious period, one can model this fact by using different values of $\beta$ for each stage. Most models of HIV infection are

2000 Mathematics Subject Classification. 34D23, 34A34, 92D30.

Key words and phrases. Nonlinear dynamical systems, epidemic models, global stability. 
with four or more stages of infection. The case of infection with carriers is also such example. This is the case of HBV infection, tuberculosis ([40]), cholera or typhoid. A second reason is that a chain of compartments gives an Erlang distribution for the residence time $[28,23,36,35]$.

Actually introducing different stages in the simple $S E I R$ model can be motivated by biological reasons or phenomenological reasons. For example in within-host models of malaria the introduction of different class of parazitised erythrocytes is sensible $[14,13]$. On the other side if the density function of residence period in a stage is measured, then a linear chain or parallel linear chains can give a good phenomenological model [24]. The SI models with different infectiousness has now a long story in the literature. The first models has been introduced for the transmission of HIV $[28,2]$. These models have been called Staged progression models (SP) in [22], where another structure is also introduced the differential infectivity models (DI).

Our models encompass the so-called staged-progression models and differential infectivity models with mass action incidence, but can also represent more complex relations between different infectious classes as for example CBPP models (Continuous Bovine Pleuro Pneumonia) [3] [45, 22]. These models represent also within-host models of parasites such that HIV or malaria plasmodium [7, 14, 13, 41]

We consider $k$ latent classes $E_{1}, \ldots, E_{k}$ and $n-k$ infectious classes $I_{k+1}, \ldots, I_{n}$. The SP models with latent classes are given by the following system.

$$
\left\{\begin{array}{l}
\dot{S}=\varphi(S)-S \sum_{i=k+1}^{n} \beta_{i} I_{i} \\
\dot{E}_{1}=S \sum_{i=k+1}^{n} \beta_{i} I_{i}-\alpha_{1} E_{1} \\
\cdots \\
\dot{E}_{k}=\gamma_{k-1} E_{k-1}-\alpha_{h} E_{k} \\
\dot{I}_{k+1}=\gamma_{k} E_{k}-\alpha_{k+1} I_{k+1} \\
\cdots \\
\dot{I}_{n}=\gamma_{n-1} I_{n-1}-\alpha_{n} I_{n} \\
\dot{R}=\gamma_{n+1} I_{n}-\alpha_{n+1} R
\end{array}\right.
$$

where $S$ is the density of susceptible individuals ( for example area density ), the function $\varphi$ is usually $\varphi(S)=\Lambda-\mu_{S} S, I_{i}$ is the density of infectious individuals of class $i$. We denote by $\alpha_{i}=\gamma_{i}+\mu_{i}$ the sum of the progression rate in the next compartment and the specific death of the $i$-th compartment. For the last compartment $\alpha_{n+1}$ is simply the death-rate, however we keep this notation for homogeneity of the formulation.

We will consider this system with a class $\mathcal{C}^{1}$ function $\varphi$ such that the system $\dot{x}=$ $\varphi(x)$ has a unique globally asymptotically stable equilibrium $x^{*}>0$ on $\mathbb{R}^{+}$. This means that the population, when there is no disease, stabilizes to a demographic equilibrium $x^{*}$.

With this hypothesis $\alpha_{i} \geq \gamma_{i}$. We remark that we do not need this assumption on the parameters. In some models, as for example in within-host models for HIV or Malaria $([14,7])$, some $\gamma_{i}$ can be greater than $\alpha_{i}$ to take in account the multiplication of parasites when leaving an infected cell. 
In these models different class of latent (infected but not infectious) individuals can also be introduced, simply in setting some $\beta_{i}$ to 0 , giving a $S E I R$ stagedprogression model.

On the contrary a DI model is a model in which individuals enter a specific group when they become infected and stay in that group until they are no longer involved in transmission.

$$
\left\{\begin{array}{l}
\dot{S}=\Lambda-\mu_{S} S-S \sum_{i=1}^{n} \beta_{i} I_{i} \\
\dot{I}_{1}=\pi_{1} S \sum_{i=1}^{n} \beta_{i} I_{i}-\alpha_{1} I_{1} \\
\cdots \\
\dot{I}_{i}=\pi_{i} S \sum_{i=1}^{n} \beta_{i} I_{i}-\alpha_{i} I_{i} \\
\cdots \\
\dot{I}_{n}=\pi_{n} S \sum_{i=1}^{n} \beta_{i} I_{i}-\alpha_{n} I_{n} \\
\dot{R}=\sum_{i=1}^{n} \gamma_{i} I_{i}-\alpha_{n} R
\end{array}\right.
$$

with $\sum_{i=1}^{n} \pi_{i}=1$

The meaning of the coefficients is the same as before.

Finally DISP models can be considered, i.e. parallel linear chains of different length. All these models can be written under one single form.

The models considered in this paper have the following structure.

$$
\left\{\begin{array}{l}
\dot{x}=\varphi(x)-x \beta^{T} . y \\
\dot{y}=\left(x \beta^{T} . y\right) \mathbf{b}+A y=\left(A+\mathbf{b} \beta^{T}\right) y
\end{array}\right.
$$

where $x \in \mathbb{R}_{+}$represents the class of susceptible individuals, $y \in \mathbb{R}_{+}^{n}$, as a column vector, represents the different class of latent, infectious and removed individuals. The different infectiousness coefficient are in $\beta \in \mathbb{R}_{+}^{k}$ a nonnegative vector. We denote by $\beta^{T}$ the transposition, hence $\beta^{T} . y$ is the inner product of $\beta$ and $y$. The vector $\mathbf{b} \in \mathbb{R}_{+}^{n}$ is nonnegative and $A$ is a stable Metzler matrix [25, 37] (A Metzler matrix is a matrix with off-diagonal entries nonnegative, some authors also call these matrices quasipositive)

The function $\varphi$ represents the demography of the population. For example $\varphi$ can be the widely used function $\varphi(x)=\Lambda-\mu_{x} x$. Hence we assume that $\varphi$ is of class $\mathcal{C}^{1}$ function and that the system $\dot{x}=\varphi(x)$ has a unique positive, globally asymptotic equilibrium $x^{*}$ on $\mathbb{R}^{+}$. In other words when there is no disease the population stabilize to a demographic equilibrium $x^{*}$. This hypothesis is usual in the literature.

There are two schools for matrices like $A$. The first one, uses Metzler matrices and is represented by J.A. Jacquez, D. Luenberger or H. Thieme [24, 25, 37, 44]. The second one uses $M$-matrices, the opposite of Metzler matrices, represented by Bermans and Plemmons or van den Driessche $([5,45])$. We choose to stick to the Jacquez point of view, natural for compartment models, since our matrix $A$ represents the exchanges between compartments (and also with the outside world). 
Or expressed differently $\dot{y}=A y$ describes the dynamic of the infected compartments when the recruitment of infected is blocked.

In this paper, we will give a simple formula for $\mathcal{R}_{0}$, prove that if $\mathcal{R}_{0} \leq 1$ then the disease free equilibrium is globally asymptotically stable, prove that if $\mathcal{R}_{0}>1$ there exists an unique endemic equilibrium. We will prove the global stability for $\mathrm{SP}$ and DI models. Theses results generalize the results obtained in $[21,22]$

2. Notations and applications. We show in this section how our structure can take into account the DI,SP, and DISP models with mass action incidence. It is the straightforward to represent more complex relation between infected (exposed and infectious) compartments.

The usual euclidean norm of a vector $x$ is denoted by $\|x\|_{2}^{2}$. The canonical basis of $\mathbb{R}^{n}$ is denoted by $\left\{e_{1}, \cdots, e_{n}\right\}$. For example $e_{1}=[1,0, \cdots, 0]^{T}$.

If $x \in \mathbb{R}^{n}$ we denote by $x_{i}$ the $i$-th component of $x$. Equivalently $x_{i}=x^{T} . e_{i}$.

For matrices $A, B$ we write $A \leq B$ if $a_{i j} \leq b_{i j}$ for all $i$ and $j, A<B$ if $A \leq B$ and $A \neq B, A \ll B$ if $a_{i j}<b_{i j}$ for all $i$ and $j$. For a matrix $A$ we denote by $A(i, j)$ the entry at the row $i$, column $j$.

$I_{n}$ denotes the $n \times n$ identity matrix. $I_{m, n}$ the $m \times n$ matrix with 1 on the diagonal. $0_{p, q}$ is the $p \times q$ zero matrix.

We also denote by $A^{-T}$ the transpose of the inverse of $A$.

For later references we precise in this section the different values of the parameters of the general system (3) for different particular system.

2.1. SP systems. The system (1) is a particular case of (3) with

$$
\begin{gathered}
A=\left[\begin{array}{ccccc}
-\alpha_{1} & 0 & 0 & \cdots & 0 \\
\gamma_{1} & -\alpha_{2} & 0 & \cdots & 0 \\
0 & \gamma_{2} & -\alpha_{3} & \cdots & 0 \\
\vdots & \ddots & \ddots & \ddots & \vdots \\
0 & \cdots & 0 & \gamma_{n} & -\alpha_{n+1}
\end{array}\right] \\
\beta=\left[\beta_{1}, \cdots, \beta_{n}, 0\right]^{T} ; \quad \mathbf{b}=e_{1}
\end{gathered}
$$

It is sufficient to consider the $k$ first components of $\beta$ equal to 0 to obtain a $S E_{1} \cdots E_{k} I_{k+1} \cdots E_{n} R$ model

2.2. DI systems. The system 2 is can be written as (3) with

$$
\begin{gathered}
A=\left[\begin{array}{ccccc}
-\alpha_{1} & 0 & 0 & \cdots & 0 \\
0 & -\alpha_{2} & 0 & \cdots & 0 \\
\vdots & \ddots & \ddots & \ddots & \vdots \\
0 & \cdots & 0 & -\alpha_{n} & 0 \\
\gamma_{1} & \cdots & \gamma_{n-2} & \gamma_{n-1} & -\alpha_{n+1}
\end{array}\right] \\
\beta=\left[\beta_{1}, \cdots, \beta_{n}, 0\right]^{T} ; \quad \mathbf{b}=\pi_{1} e_{1}+\cdots+\pi_{n} e_{n}
\end{gathered}
$$

2.3. DISP systems. Similarly we can define DISP models of $k$ parallel linear chains of different lengths. Introducing dummy class we can suppose that the length of the parallel chains are equal. Then the system can be written under the general form (3) with $A$ a diagonal bloc matrix $A=\operatorname{diag}\left(A_{1}, \cdots, A_{k}\right.$ where each $A_{i}$ is a matrix similar to $A$ defined in section 2.1 , and if we identify the state space of the system with $\mathbb{R} \times\left(\mathbb{R}^{n}\right)^{k}$ the vector $\mathbf{b}$ is

$$
\mathbf{b}=\pi_{1} e_{1}+\cdots+\pi_{n} e_{n}
$$


where $e_{i}$ is the first vector of the canonical basis of the $i$-th component of $\left(\mathbb{R}^{n}\right)^{k}$.

3. Dissipativity and global stability of the DFE. We consider the general structured model (3). We only assume that the coefficients are nonnegative. We denote by $x^{*}$ the global asymptotic equilibrium of $\varphi$ on $\mathbb{R}$. This is the demographic equilibria of the population without disease. The disease free equilibrium is then $\left(x^{*}, 0, \cdots, 0\right)$.

3.1. Positive Invariance of the nonnegative orthant. With the hypothesis on the parameters of (3) it is straightforward to check the positive invariance of the nonnegative orthant $\mathbb{R}_{+}^{n+1}$ by this system.

3.2. Boundedness and dissipativity. We will prove that there always exits a convex compact positively invariant absorbing set $K_{\rho}$ for the system (3).

Since $A$ is Metzler stable matrix (equivalently $-A$ is a $M$-matrix [5]) there exists a positive vector $c \gg 0$ such that $A^{T} c \ll 0$. We define

$$
V_{B}(x, y)=c^{T} \cdot \mathbf{b} x+c^{T} y
$$

Since $c \gg 0$ we have $c . \mathbf{b}^{T} \gg 0$.The derivative $\dot{V}_{B}$ of $V_{B}$ along the trajectories of (3) is

$$
\begin{aligned}
\dot{V}_{B} & =c^{T} \cdot \mathbf{b} \varphi(x)-x c^{T} \cdot \mathbf{b} \beta^{T} \cdot y+x c^{T} \cdot \mathbf{b} \beta^{T} \cdot y+c^{T} \cdot A y \\
& =c^{T} \cdot \mathbf{b} \varphi(x)+c^{T} \cdot A y
\end{aligned}
$$

We set $\Phi=\max _{x \in \mathbb{R}_{+}} \varphi(x)$ and we define

$$
\delta=\frac{\|c\|_{2} c^{T} \cdot \mathbf{b} \Phi}{\min _{i}\left(-A^{T} c\right)_{i}}+c^{T} \cdot \mathbf{b} x^{*}
$$

We now consider for $\rho \geq \delta$ the set $K_{\rho}$ defined by

$$
\left.K_{\rho}=V_{B}^{-1}[0, \rho]\right) \cap \mathbb{R}_{+}^{n+1}
$$

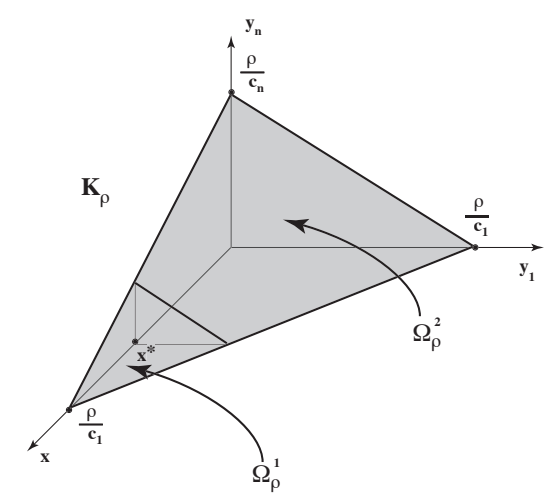

FIGURE 1. simplex

$K_{\rho}$ is a compact set of the nonnegative orthant which contain $\left[x^{*}, 0, \cdots, 0\right]^{T}$ in its interior. Actually $K_{\rho}$ is the $n+1$-dimensional simplex. We will prove that $K_{\rho}$ is a compact positively invariant set for (3) if $\rho \geq \delta$. Since the nonnegative orthant 
is positively invariant it is sufficient to prove that no trajectory of (3) can leave $K_{\rho}$ by its boundary $\Omega_{\rho}$ where

$$
\left.\Omega_{\rho}=V^{-1}\{\rho\}\right) \cap \mathbb{R}_{+}^{n+1}
$$

This is equivalent to prove that $\dot{V} \leq 0$ on $\Omega_{\rho}$. For this we divide $\Omega_{\rho}$ in two parts

$$
\Omega_{\rho}^{1}=\Omega_{\rho} \cap\left\{(x, y) \in \mathbb{R}_{+}^{n+1} \mid x \geq x^{*}\right\}
$$

and

$$
\Omega_{\rho}^{1}=\Omega_{\rho} \cap\left\{(x, y) \in \mathbb{R}_{+}^{n+1} \mid x^{*} \geq x\right\}
$$

Since $x^{*}$ is globally asymptotically stable, on $\Omega_{\rho}^{1}, \varphi(x) \leq 0$ and by (4), we have $\dot{V} \leq 0$ on $\Omega_{\rho}^{1}$.

By definition of $\Omega_{\rho}$ on $\Omega_{\rho}^{2}$ we have $V_{B}(x, y)=\rho=c^{T} \mathbf{b} x+c^{T} y$.

By Cauchy-Schwarz inequality, on $\Omega_{\rho}^{2}$ we have

$$
\|c\|_{2}\|y\|_{2} \geq\left\langle c^{T} \mid y\right\rangle=\rho-c^{T} \cdot \mathbf{b} x \geq \delta-c^{T} \cdot \mathbf{b} x^{*}=\frac{\|c\|_{2} c^{T} \cdot \mathbf{b} \Phi}{\min _{i}\left(-A^{T} c\right)_{i}}
$$

Which implies

$$
\|y\|_{2} \geq \frac{c^{T} \mathbf{b} \Phi}{\min _{i}\left(-A^{T} c\right)_{i}}
$$

Therefore on $\Omega_{\rho}^{2}$ the following inequalities are satisfied

$$
-c^{T} \cdot A y \geq \min _{i}\left(-A^{T} c\right)_{i}\left(y_{1}+\cdots+y_{n}\right) \geq \min _{i}\left(-A^{T} c\right)_{i}\|y\|_{2} \geq c^{T} \cdot \mathbf{b} \Phi
$$

Hence

$$
\dot{V}_{B}=c^{T} \cdot \mathbf{b} \varphi(x)+c^{T} \cdot A y \leq c^{T} \cdot \mathbf{b} \Phi+c^{T} \cdot A y \leq 0
$$

on $\Omega_{\rho}^{2}$.

This ends the proof of the positive invariance of $\Omega_{\rho}$ for $\rho \geq \delta$.

Since $V(x, y) \rightarrow+\infty$ when $(x, y) \rightarrow+\infty$, any initial condition $\left(x_{0}, y_{0}\right)$ in the nonnegative orthant is contained in a $K_{\rho}$. This proves that any trajectory is forward bounded.

We will now prove that $K_{\rho}$ is a compact absorbing set for any $\rho>\delta$. An absorbing set $D$ is a neighborhood such that a trajectory of the system starting from any initial condition enters and remains in $D$ for a sufficiently large time $T$. Let consider an initial condition $\left(x_{0}, y_{0}\right)$ and the forward trajectory from this initial condition. We assume $\left(x_{0}, y_{0}\right) \notin K_{\rho}$ otherwise since we have proved that $K_{\rho}$ is positively invariant we are finished. We will prove by contradiction that this trajectory enters $K_{\rho}$. We suppose, by contradiction, that the trajectory stays always out of $K_{\rho}$. In other words for any points $(x, y)$ of the trajectory $V(x, y)>\rho>\delta$. An analogous computation as the preceding one implies $\dot{V} \leq 0$. The function $V$ is decreasing on the trajectory. Now since any trajectory is bounded the set $\Omega_{x_{0}, y_{0}}$ of $\omega$-limit points of the trajectory is non empty and by hypothesis is contained in the closure of the complementary set of $K_{\rho}$, the set $\overline{\left\{(x, y) \notin K_{\rho}\right\}}$. By LaSalle's invariance principle $\Omega_{x_{0}, y_{0}} \subset\{\dot{V}=0\} \cap \overline{\left\{(x, y) \notin K_{\rho}\right\}}$. Since $\dot{V}(x, y)=c^{T} . \beta \varphi(x)+c^{T}$.Ay we have $\{\dot{V}=0\}=\left\{\left(x^{*}, 0, \cdots, 0\right\}\right.$. The point $\left(x^{*}, 0, \cdots, 0\right)$ is in the interior of $K_{\rho}$, this implies that $\Omega_{x_{0}, y_{0}}=\emptyset$, hence a contradiction. 
3.3. Basic reproduction ratio. As usual the basic reproduction number is the expected number of secondary cases produced in a completely susceptible population, by a typical infected individual during its entire period of infectiousness $[17,45,9,8]$. We can give a simple elegant formula for the $\mathcal{R}_{0}$ (compare with $[22,38])$.

To obtain $\mathcal{R}_{0}$ we can use the techniques developed in [45]. We claim

$$
\mathcal{R}_{0}=x^{*} \beta^{T}\left(-A^{-1}\right) \mathbf{b}
$$

We use the expression $\left(-A^{-1}\right)$ to put the emphasis on the fact that $\left(-A^{-1}\right)>0$ because $A$ is Metzler stable.

Using the frame of [45], we define by $\mathcal{F}_{i}(x, y)$ the rate of appearance of new infections in compartment $i$, and by $\mathcal{V}_{i}(x, y)$ the rate of transfer of individuals in and out the compartment $i$ by all other means. The matrix $\mathcal{V}$ is the "mass" balance of the compartments. Note that our $\mathcal{V}$ is the opposite of the same used in [45]. Then

$$
\mathcal{F}(x, y)=\left[\begin{array}{c}
0 \\
x \beta^{T} . y \mathbf{b}
\end{array}\right]
$$

and

$$
\mathcal{V}(x, y)=\left[\begin{array}{c}
\varphi(x)-x \beta^{T} \cdot y \\
A y
\end{array}\right]
$$

The Jacobian matrices are

$$
D \mathcal{F}(x, y)=\left[\begin{array}{cc}
0 & 0 \\
\beta^{T} . y \mathbf{b} & x \mathbf{b} \beta^{T}
\end{array}\right] \quad D \mathcal{V}(x, y)=\left[\begin{array}{cc}
\varphi^{\prime}(x)-\beta^{T} . y & -x \beta^{T} \\
0 & A
\end{array}\right]
$$

Noting that we have sorted the variables in the reverse order of [45], we set $F=x^{*} \mathbf{b} \beta^{T}$ an $V=A$

It is proved in [45] that the basic reproduction number is the spectral radius of the next generation matrix for the model, namely $-F V^{-1}$ (the minus sign comes from Metzler matrices used in place of $M$-matrices)

$$
\mathcal{R}_{0}=\rho\left(-F V^{-1}\right)=\rho\left(-x^{*} \mathbf{b} \beta^{T} A^{-1}\right)
$$

It is clear that $-x^{*} \mathbf{b} \beta^{T} A^{-1}$ is a rank one matrix, the only nonzero eigenvalue is given by $-x^{*} \beta^{T} A^{-1} \mathbf{b}$, which exactly our claim.

3.4. Equilibria. We will also obtain a simple formula for the equilibria.

There exists an evident equilibrium for $(3)$ which is $\left(x^{*}, 0, \cdots, 0\right)$. We call this equilibrium the disease free equilibrium. We also denote this equilibrium by DFE. Any equilibrium $(\bar{x}, \bar{y})$ satisfies

$$
\begin{gathered}
\varphi(\bar{x})=\bar{x} \beta^{T} \bar{y} \\
\bar{x} \beta^{T} \bar{y} \mathbf{b}=A \bar{y}
\end{gathered}
$$

which gives $\bar{y}=\bar{x} \beta^{T} \bar{y}\left(-A^{-1}\right) \mathbf{b}$ and consequently

$$
\beta^{T} \bar{y}=\bar{x} \beta^{T} \bar{y} \beta^{T}\left(-A^{-1}\right) \mathbf{b}
$$

If $\beta^{T} \bar{y}=0$, since $A$ is stable, hence nonsingular, we get $\bar{y}=0$ and $\bar{x}=x^{*}$ which is the DFE. The other case $\beta^{T} \bar{y} \neq 0$ gives

$$
\bar{x}=\frac{1}{\beta^{T}\left(-A^{-1}\right) \mathbf{b}}=\frac{x^{*}}{\mathcal{R}_{0}}
$$

and

$$
\bar{y}=\varphi(\bar{x})\left(-A^{-1}\right) \mathbf{b}
$$


Since $x^{*}$ is globally asymptotically stable $\varphi(\bar{x})$ is positive if and only if $\bar{x}<x^{*}$, hence if and only if $\mathcal{R}_{0}>1$. Using that $A$ is a Metzler stable matrix we deduce $\bar{y}>0$ if and only if $\mathcal{R}_{0}>1$.

We have proved that a unique endemic equilibrium exists (in the nonnegative orthant) if and only $\mathcal{R}_{0}>1$. This endemic equilibrium is in the compact absorbing set $K_{\rho}$

4. Global asymptotic stability of the DFE. We give a simple proof for the system (3) with a general stable Metzler matrix $A$

The system (3) is globally asymptotically stable at the disease free equilibrium (DFE) $\left(x^{*}, 0, \cdots, 0\right)$ if and only if $\mathcal{R}_{0}=\beta^{T}\left(-A^{-1}\right) \mathbf{b} x^{*} \leqslant 1$.

Proof: In a first step we will prove that if $\mathcal{R}_{0} \leqslant 1$, the DFE is globally asymptotically stable on nonnegative orthant. It is well know that if $\mathcal{R}_{0}>1$ then the DFE is unstable. Thus the condition $\mathcal{R}_{0} \leqslant 1$ is necessary.

To prove the sufficiency we consider the following Lyapunov function (in LaSalle's sense $[30,31])$ defined on the positive orthant.

$$
V_{D F E}(x, y)=\frac{1}{x^{*}}\left(x-x^{*} \ln x\right)-\beta^{T} A^{-1} y-\frac{1}{x^{*}}\left(x^{*}-x^{*} \ln x^{*}\right)
$$

This function is nonnegative in general, since we only knows that $\beta^{T} A^{-1}>0$.

It's time derivative along the trajectories of system (3) is

$$
\dot{V}_{D F E}=\frac{1}{x^{*}}\left[\frac{x-x^{*}}{x} \varphi(x)-x \beta^{T} y+x^{*} \beta^{T} y\right]-x \beta^{T} y \beta^{T} A^{-1} \mathbf{b}-\beta^{T} y
$$

simplifying and using the expression for $\mathcal{R}_{0}$ we obtain

$$
\dot{V}=\frac{1}{x^{*}}\left[\frac{x-x^{*}}{x} \varphi(x)-x \beta^{T} y+\right]+x \beta^{T} y \frac{\mathcal{R}_{0}}{x^{*}}
$$

or equivalently

$$
\dot{V}=\frac{x-x^{*}}{x^{*} x} \varphi(x)+\frac{x}{x^{*}} \beta^{T} y\left(\mathcal{R}_{0}-1\right)
$$

With the hypothesis that $x^{*}$ is globally asymptotically stable on $\mathbb{R}_{+}$for the system $\dot{x}=\varphi(x)$, we have $\left(x-x^{*}\right) \varphi(x) \leqslant 0$ for all $x \geqslant 0$. Therefore $\dot{V} \leqslant 0$ for all $(x, y)$ in the positive orthant. We restrict our attention, for the moment, to the positively invariant compact set $K_{\rho}$. The attractivity of the DFE follows from LaSalle invariance principle since the largest invariant set contained in $\{(x, y) \in$ $\left.K_{\rho} \mid \dot{V}=0\right\}$ is reduced to the DFE. This proves the global asymptotic stability on $K_{\rho}([6]$, Theorem 3.7.11, page 346$)$. Since $K_{\rho}$ is absorbing this proves the global asymptotic stability on the nonnegative orthant.

5. Global stability of the endemic equilibrium. Global results of stability for the DFE as well for the endemic equilibrium for epidemic models are not so common $[17,44]$. Global stability results for the endemic equilibrium using the Li-Muldowney techniques ([33]) bear upon properties of monotone systems. Usually the PoincaréBendixson property of monotone systems in dimension 3 is used. [15, 33, 40]. These techniques are far from being straightforward for high dimensional systems. In recent years Lyapunov methods have been used. A Volterra-like Lyapunov function has been used in [29] to prove global stability of the endemic equilibrium for SEIR models. This function has a long history of application to Lotka-Volterra models [12] and was originally discovered by Volterra himself, although he did not use 
the vocabulary and the theory of Lyapunov functions. Since epidemic models are "Lotka-Volterra" like models, the pertinence of this function is not surprising. The global stability for DI models, with any number of compartments, with mass action incidence has been proved in [38]. For the SP model only local results are known. We will use a Volterra-like Lyapunov function. The difficulty is in the choice of the coefficients and in proving the negative definiteness of the derivative. We propose a general technique for finding the coefficients. The proof of the negativity is more involved and bear upon additional properties of $A$.

In this section we will describe a method to prove the global stability of the endemic equilibrium. We will apply this method to the SP model with mass action incidence, to establish the global stability, which improve results of $[22,21]$

5.1. The general stategy. The proof is based on a Volterra-like Lyapunov function, defined on the positive orthant

$$
V_{E E}(x, y)=a_{0}(x-\bar{x} \ln x)+\sum_{i=1}^{n} a_{i}\left(y_{i}-\bar{y}_{i} \ln y_{i}\right)+K
$$

Where $K$ is the constant $K=a_{0}(\bar{x}-\bar{x} \ln \bar{x})+\sum_{i=1}^{n} a_{i}\left(\bar{y}_{i}-\bar{y}_{i} \ln \bar{y}_{i}\right)$. This function is positive on the positive orthant.

We first prove that we can choose the coefficients such that, in the expression of $\dot{V}_{E E}$, there are no linear terms in $y$ and no bilinear terms.

It is sufficient to show that, if we set $a=\left(a_{1}, \cdots, a_{n}\right)$, there is a nonnegative solution of

$$
\left[\begin{array}{cc}
-1 & \mathbf{b}^{T} \\
\bar{x} \beta & A^{T}
\end{array}\right]\left[\begin{array}{c}
a_{0} \\
a
\end{array}\right]=0
$$

Since

$$
\operatorname{det}\left[\begin{array}{cc}
-1 & \mathbf{b}^{T} \\
\bar{x} \beta & A^{T}
\end{array}\right]=-1+\mathbf{b}^{T}\left(-A^{-T}\right) \bar{x} \beta=-1-\beta^{T} A^{-1} \mathbf{b}=-1+1=0
$$

using the relation (7) for $\bar{x}$

The matrix is a codimension 1 matrix, the kernel is one dimensional, then we have one degree of freedom with $a=-a_{0} \bar{x} A^{-T} \beta$. Any positive value for $a_{0}$ gives a nonnegative $a$. We choose.

With this choice, denoting $\operatorname{diag}(a)$ the diagonal matrix with elements of $a$ on the diagonal

$$
\begin{aligned}
\dot{V}_{E E}= & a_{0} \frac{x-\bar{x}}{x} \varphi(x)-a_{0} x \beta^{T} y+\underline{a_{0} \bar{x} \beta^{T} y}+ \\
& x \beta^{T} y a^{T} \mathbf{b}+a^{T} A y \\
& -x \beta^{T} y a^{T} \operatorname{diag}(\bar{y})(\operatorname{diag}(y))^{-1} \mathbf{b}-a^{T} \operatorname{diag}(\bar{y})(\operatorname{diag}(y))^{-1} A y
\end{aligned}
$$

Since $a_{0}=\mathbf{b}^{T} a=a^{T} \mathbf{b}$ and $a=a_{0} \bar{x}\left(-A^{-T}\right) \beta$ the terms $-a_{0} x \beta^{T} y$ and $x \beta^{T}$ y $a^{T} \mathbf{b}$ (respectively $a_{0} \bar{x} \beta^{T} y$ and $a^{T} A y$ ) cancel.

Which gives

$$
\dot{V}_{E E}=a_{0} \frac{x-\bar{x}}{x} \varphi(x)-x \beta^{T} y a^{T} \operatorname{diag}(\bar{y})(\operatorname{diag}(y))^{-1} \mathbf{b}-a^{T} \operatorname{diag}(\bar{y})(\operatorname{diag}(y))^{-1} A y
$$

The problem is now to rewrite the last expressions. 
5.2. The SP model. We will apply the general strategy to a SP model with latent classes. Then we consider a $S E_{1} \cdots E_{k} I_{k+1} \cdots I_{n}$ model, and we prove the global asymptotic stability of the endemic equilibrium when $\mathcal{R}_{0}>1$ for the usual demographic function $\Lambda-\mu_{X} x$ and give a sufficient condition for global asymptotic stability in the general case. If we remark that in this model, the latent classes can be considered as infected classes with 0 transmission, i.e. $\beta_{i}=0$ for $i=1, \cdots, k$.

Theorem 1. For the system (1) when $\mathcal{R}_{0}>1$ there is a unique endemic equilibrium. This endemic is then globally asymptotically stable for the function $\varphi(S)=\Lambda-$ $-\mu_{S} S$. More generally for a class $\mathcal{C}^{1}$ function $\varphi$, if $\max \varphi^{\prime} \leq \frac{\beta_{1}}{\alpha_{1}} \varphi\left(\frac{x^{*}}{\mathcal{R}_{0}}\right)$ the endemic equilibrium is globally asymptotically stable.

We consider the equations defining the endemic equilibrium.

$$
\begin{aligned}
& \varphi(\bar{x})=\left(\sum_{i=1}^{n} \beta_{i} \bar{I}_{i}\right) \bar{x} \\
& \left(\sum_{i=1}^{n} \beta_{i} \bar{I}_{i}\right) \bar{x}=\alpha_{1} \bar{I}_{1} \\
& \gamma_{1} \bar{I}_{1}=\alpha_{2} \bar{I}_{2} \\
& \gamma_{2} \bar{I}_{2}=\alpha_{3} \bar{I}_{3} \\
& \ldots \ldots \ldots . . \\
& \gamma_{n-1} \bar{I}_{n-1}=\alpha_{n} \bar{I}_{n}
\end{aligned}
$$

The relation (11) between the coefficients can be developed in

$$
\left\{\begin{array}{l}
b_{1}-a=0 \\
a \beta_{1} \bar{x}-b_{1} \alpha_{1}+b_{2} \gamma_{1}=0 \\
a \beta_{2} \bar{x}-b_{2} \alpha_{2}+b_{3} \gamma_{2}=0 \\
\ldots \ldots \ldots \\
a \beta_{n} \bar{x}-b_{n} \alpha_{n}=0
\end{array}\right.
$$

We deduce for the endemic equilibrium

$$
\begin{aligned}
b_{1} \alpha_{1} \bar{I}_{1} & =a \beta_{1} \bar{x} \bar{I}_{1}+b_{2} \gamma_{1} \bar{I}_{1} \\
& =a \beta_{1} \bar{x} \bar{I}_{1}+b_{2} \alpha_{2} \bar{I}_{2} \\
& =a \beta_{1} \bar{x} \bar{I}_{1}+a \beta_{2} \bar{x} \bar{I}_{2}+b_{3} \alpha_{3} \bar{I}_{3} \\
& \ldots \ldots \ldots+\cdots \\
& =a \beta_{1} \bar{x} \bar{I}_{1}+a \beta_{2} \bar{x} \bar{I}_{2}+\cdots+a \beta_{n} \bar{x} \bar{I}_{n}
\end{aligned}
$$

More generally we have

$$
\begin{aligned}
b_{i} \alpha_{i} \bar{I}_{i} & =a \beta_{i} \bar{x} \bar{I}_{i}+\cdots+a \beta_{n} \bar{x} \bar{I}_{n} \\
& =\sum_{j=i}^{n} a \beta_{j} \bar{x} \bar{I}_{j}
\end{aligned}
$$


and

$$
\begin{aligned}
b_{i+1} \gamma_{i} \bar{I}_{i} & =a \beta_{i+1} \bar{x} \bar{I}_{i+1}+\cdots+a \beta_{n} \bar{x} \bar{I}_{n} \\
& =\sum_{j=i+1}^{n} a \beta_{j} \bar{x} \bar{I}_{j}
\end{aligned}
$$

The derivative of $V_{E E}$ expressed in (12) is

$$
\dot{V}=a \varphi(x)\left(1-\frac{\bar{x}}{x}\right)+\sum_{i=1}^{n} b_{i} \alpha_{i} \bar{I}_{i}-a \beta_{1} \bar{x} \bar{I}_{1} \frac{x}{\bar{x}}-\sum_{i=2}^{n} a \beta_{i} \bar{x} \bar{I}_{i} \frac{\bar{I}_{1}}{I_{1}} \frac{x}{\bar{x}} \frac{I_{i}}{\bar{I}_{i}}-\sum_{i=1}^{n-1} \gamma_{i} b_{i+1} \bar{I}_{i} \frac{I_{i}}{\bar{I}_{i}} \frac{\bar{I}_{i+1}}{I_{i+1}}
$$

But we can also write

$$
\sum_{i=1}^{n} b_{i} \alpha_{i} \bar{I}_{i}=\sum_{j=1}^{n} a j \beta_{j} \bar{x} \bar{I}_{j}
$$

Then

$$
\begin{aligned}
\sum_{i=1}^{n-1} \gamma_{i} b_{i+1} \bar{I}_{i} \frac{I_{i}}{\bar{I}_{i}} \frac{\bar{I}_{i+1}}{I_{i+1}} & =\sum_{i=1}^{n-1}\left(\sum_{j=i+1}^{n} a \beta_{j} \bar{x} \bar{I}_{j}\right) \frac{I_{i}}{\bar{I}_{i}} \frac{\bar{I}_{i+1}}{I_{i+1}} \\
& =\sum_{j=2}^{n} a \beta_{j} \bar{x} \bar{I}_{j}\left(\sum_{i=1}^{j-1} \frac{I_{i}}{\bar{I}_{i}} \frac{\bar{I}_{i+1}}{I_{i+1}}\right)
\end{aligned}
$$

rearranging this equation gives

$$
\begin{aligned}
\dot{V}= & a \varphi(x)\left(1-\frac{\bar{x}}{x}\right)+\sum_{j=1}^{n} a j \beta_{j} \bar{x} \bar{I}_{j}-a \beta_{1} \bar{x} \bar{I}_{1} \frac{x}{\bar{x}} \\
& -\sum_{j=2}^{n} a \beta_{j} \bar{x} \bar{I}_{j} \frac{\bar{I}_{1}}{I_{1}} \frac{x}{\bar{x}} \frac{I_{j}}{\bar{I}_{j}}-\sum_{j=2}^{n} a \beta_{j} \bar{x} \bar{I}_{j}\left(\sum_{i=1}^{j-1} \frac{I_{i}}{\bar{I}_{i}} \frac{\bar{I}_{i+1}}{I_{i+1}}\right)
\end{aligned}
$$

or equivalently

$$
\begin{aligned}
\dot{V}= & a \varphi(x)\left(1-\frac{\bar{x}}{x}\right)+a \beta_{1} \bar{x} \bar{I}_{1}-a \beta_{1} \bar{x} \bar{I}_{1} \frac{x}{\bar{x}} \\
& +\sum_{j=2}^{n} a \beta_{j} \bar{x} \bar{I}_{j}\left[j-\frac{\bar{I}_{1}}{I_{1}} \frac{x}{\bar{x}} \frac{I_{j}}{\bar{I}_{j}}-\sum_{i=1}^{j-1} \frac{I_{i}}{\bar{I}_{i}} \frac{\bar{I}_{i+1}}{I_{i+1}}\right]
\end{aligned}
$$

If we group the terms in brakets in order to have the geometrical mean equal to 1 , we will have

$$
\begin{aligned}
\dot{V}= & a \varphi(x)\left(1-\frac{\bar{x}}{x}\right)+a \beta_{1} \bar{x} \bar{I}_{1}\left(1-\frac{x}{\bar{x}}\right)+\sum_{j=2}^{n} a \beta_{j} \bar{x} \bar{I}_{j}\left(\frac{\bar{x}}{x}-1\right) \\
& \left.+\sum_{j=2}^{n} a \beta_{j} \bar{x} \bar{I}_{j}\left[(j+1)-\frac{\bar{x}}{x}-\frac{\bar{I}_{1}}{I_{1}} \frac{x}{\bar{x}} \frac{I_{j}}{\bar{I}_{j}}-\sum_{i=1}^{j-1} \frac{I_{i}}{\bar{I}_{i}} \frac{\bar{I}_{i+1}}{I_{i+1}}\right)\right]
\end{aligned}
$$

But we also know that

$$
a \varphi(\bar{x})=a \alpha_{1} \bar{I}_{1}=\sum_{j=1}^{n} a \beta_{j} \bar{x} \bar{I}_{j}
$$


So

$$
\begin{aligned}
\dot{V}= & a \varphi(x)\left(1-\frac{\bar{x}}{x}\right)+a \varphi(\bar{x})\left(\frac{\bar{x}}{x}-1\right)+a \beta_{1} \bar{x} \bar{I}_{1}\left(2-\frac{x}{\bar{x}}-\frac{\bar{x}}{x}\right) \\
& \left.+\sum_{j=2}^{n} a \beta_{j} \bar{x} \bar{I}_{j}\left[(j+1)-\frac{\bar{x}}{x}-\frac{\bar{I}_{1}}{I_{1}} \frac{x}{\bar{x}} \frac{I_{j}}{\bar{I}_{j}}-\sum_{i=1}^{j-1} \frac{I_{i}}{\bar{I}_{i}} \frac{\bar{I}_{i+1}}{I_{i+1}}\right)\right]
\end{aligned}
$$

we have

$a \varphi(x)\left(1-\frac{\bar{x}}{x}\right)+a \varphi(\bar{x})\left(\frac{\bar{x}}{x}-1\right)+a \beta_{1} \bar{x} \bar{I}_{1}\left(2-\frac{x}{\bar{x}}-\frac{\bar{x}}{x}\right)=a \frac{x-\bar{x}}{x}\left[\varphi(x)-\varphi(\bar{x})-\beta_{1} \bar{x} \bar{I}_{1} \frac{x-\bar{x}}{x}\right]$

By observing that $\varphi(x)=\varphi(\bar{x})+(x-\bar{x}) \varphi^{\prime}(c)$ where $\left.c \in\right] \bar{x}, x[$, we have

$$
\begin{aligned}
\dot{V}= & a \frac{(x-\bar{x})^{2}}{x}\left[\varphi^{\prime}(c)-\beta_{1} \bar{I}_{1}\right] \\
& \left.+\sum_{j=2}^{n} a \beta_{j} \bar{x} \bar{I}_{j}\left[(j+1)-\frac{\bar{x}}{x}-\frac{\bar{I}_{1}}{I_{1}} \frac{x}{\bar{x}} \frac{I_{j}}{\bar{I}_{j}}-\sum_{i=1}^{j-1} \frac{I_{i}}{\bar{I}_{i}} \frac{\bar{I}_{i+1}}{I_{i+1}}\right)\right]
\end{aligned}
$$

Then we have $\dot{V} \leqslant 0$ if and only if $\varphi^{\prime}(c)-\beta_{1} \bar{I}_{1} \leqslant 0$; but we already have $\alpha_{1} \bar{I}_{1}=\varphi(\bar{x})$ So the condition for the $\dot{V}$ to be definite negative is

$$
\dot{V} \leqslant 0 \quad \text { iff } \quad \varphi^{\prime}(c) \leqslant \frac{\beta_{1}}{\alpha_{1}} \varphi(\bar{x})
$$

which is obviously satisfied for $\varphi(x)=\Lambda-\mu_{x} x$.

Remark 1. This proof establishes a sligthly more general result and gives a sufficient condition for more general demographic function $\varphi(x)$

6. Conclusion. In this paper we propose a class of epidemiological systems with mass action incidence with a general structure. We provide a very simple formula for the basic reproduction ratio $\mathcal{R}_{0}$. When $\mathcal{R}_{0} \leq 1$ we prove the global asymptotic stability for the disease free equilibrium. When $\mathcal{R}_{0}>1$ we prove the uniqueness of an endemic equilibrium and provide a simple explicit formula for the components of the endemic equilibrium. Since our class of systems encompass the DI,SP and DISP models we improves the results of $[21,22,20]$ We propose a strategy for proving the global stability and apply it to SP model. The result is new.

The authors wants to thanks anonymous referee whose suggestions have improved the manuscript. The results in this paper have been exposed in june 2006 at the AIMS conference in Poitiers. We recently learned that similar results have been obtained in [16]. However our result is more general and simpler.

\section{REFERENCES}

[1] R. M. Anderson and R. M. May, "Infectious Diseases of Humans. Dynamics and Control," Oxford science publications, 1991.

[2] R. M. Anderson, R. M. May, and A. R. McLean, Possible demographic consequences of aids in developing countries., Nature, 332 (1988), 228-234.

[3] T. Balenghien, K. Chalvet-Monfray, M. Lesnoff, F. Thiaucourt, P. Sabatier, and D. Bicout, Time-delay dynamics for contagious bovine pleuropneumonia, Acta Biotheorica, (2004).

[4] E. Beretta and Y. Takeuchi, Global stability of Lotka-Volterra diffusion models with continuous time delay., SIAM J. Appl. Math., 48 (1988), 627-651.

[5] A. Berman and R. J. Plemmons, "Nonnegative matrices in the mathematical sciences," SIAM, 1994.

[6] N. P. Bhatia and G. P. Szegö, "Stability Theory of Dynamical Systems," Springer-Verlag, 1970. 
[7] P. De Leenheer and H. L. Smith, Virus dynamics: A global analysis., SIAM J. Appl. Math., 63 (2003), 1313-1327.

[8] O. Diekmann, J. A. P. Heesterbeek, and J. A. J. Metz, On the definition and the computation of the basic reproduction ratio $R_{0}$ in models for infectious diseases in heterogeneous populations, J. Math. Biol., 28 (1990), 365-382.

[9] — Mathematical epidemiology of infectious diseases, Wiley Series in Mathematical and Computational Biology, John Wiley \& Sons Ltd., Chichester, 2000. Model building, analysis and interpretation.

[10] E.H Elbasha AND A.B. Gumel, Theoretical assesment of public health impact of imperfect prophylactic hiv-1 vaccines with therapeutic benefits, J. Theoret. Biol., 68 (2006), pp. 577-614.

[11] Meng Fan, Michael Y. Li, and Ke Wang, Global stability of an SEIS epidemic model with recruitment and a varying total population size, Math. Biosci., 170 (2001), 199-208.

[12] B. S. Goh, Global stability in many-species systems, Amer. Naturalist, (1977), 135-143.

[13] M. B. Gravenor, A. L. Lloyd, P. G Kremsner, M. A. Missinou, M. English, K. Marsh, and D. Kwiatkowski, A model for estimating total parasite load in falciparum malaria patients., J Theor Biol, 217 (2002), 137-48.

[14] M. B. Gravenor, M. B. van Hensbroek, and D. Kwiatkowski, Estimating sequestered parasite population dynamics in cerebral malaria., Proc Natl Acad Sci U S A, 95 (1998), 7620-4.

[15] A.B. Gumel, C. C. McCluskey, and P. van den Driessche, Mathematical study of a stagedprogression hiv model with imperfect vaccine, J. Theoret. Biol., 68 (2006), 2105-2128.

[16] H. Guo and M.Y. Li, Global dynamics of a staged progression model for infectious diseases, Math. Biosci. Eng., 3 (2006).

[17] H. W. Hethcote, The mathematics of infectious diseases, SIAM Rev., 42 (2000), 599-653 (electronic).

[18] Herbert W. Hethcote and Horst R. Thieme, Stability of the endemic equilibrium in epidemic models with subpopulations, Math. Biosci., 75 (1985), 205-227.

[19] J. M. Hyman, J. Li, and E. A. Stanley, The initialization and sensitivity of multigroup models for the transmission of hiv, J. Theoret. Biol., 208 (2001), 227-249.

[20] J. M. Hyman AND J. LI, The reproductive number for an HIV model with differential infectivity and staged progression., Linear Algebra Appl., 398 (2005), 101-116.

[21] —, Differential susceptibility and infectivity epidemic models, Math. Biosci. Eng., 3 (2006).

[22] J. M. Hyman, J. Li, and E.A. Stanley, The differential infectivity and staged progression models for the transmission of HIV., Math. Biosci., 155 (1999), 77-109.

[23] J. A. Jacquez, "Compartmental analysis in Biology and Medicine," BioMedware, 1996.

[24] _ "Modeling with compartments," BioMedware, 1999.

[25] J. A. Jacquez and C. P. Simon, Qualitative theory of compartmental systems, SIAM Rev., 35 (1993), 43-79.

[26] J. A. Jacquez, C. P. Simon, and J.S. Koopman, The reproduction number in deterministic models of contagious diseases, Comment. Theor. Biol., 2 (1991).

[27] John A. Jacquez, Carl P. Simon, and J. Koopman, Core groups and the r0s for subgroups in heterogeneous sis and si models, in "Epidemics models : their structure and relation to data," D.Mollison, ed., Cambridge University Press, 1996, 279-301.

[28] J. A. Jacquez, C. P. Simon, J.S. Koopman, L. Sattenspiel, and T. Perry, Modeling and analyzing HIV transmission: the effect of contact patterns, Math. Biosci., 92 (1988).

[29] A. Korobeinikov and P.K Maini, A Lyapunov function and global properties for SIR and SEIR epidemiolgical models with nonlinear incidence, Math. Biosci. Eng., 1 (2004), 57-60.

[30] J. P. LaSalle, Stability theory for ordinary differential equations. stability theory for ordinary differential equations, J. Differ. Equations, 41 (1968), pp. 57-65.

[31] J. P. LaSalle, The stability of dynamical systems, Society for Industrial and Applied Mathematics, Philadelphia, Pa., 1976. With an appendix: "Limiting equations and stability of nonautonomous ordinary differential equations" by Z. Artstein, Regional Conference Series in Applied Mathematics.

[32] Michael Y. Li, John R. Graef, Liancheng Wang, and János Karsai, Global dynamics of a SEIR model with varying total population size, Math. Biosci., 160 (1999), 191-213.

[33] Michael Y. Li and James S. Muldowney, Global stability for the SEIR model in epidemiology, Math. Biosci., 125 (1995), 155-164.

[34] Michael Y. Li, Hal L. Smith, and Liancheng Wang, Global dynamics an SEIR epidemic model with vertical transmission, SIAM J. Appl. Math., 62 (2001), 58-69 (electronic). 
[35] A. L. Lloyd, Destabilization of epidemic models with the inclusion of realistic distributions of infectious periods., Proc R Soc Lond B Biol Sci, 268 (2001), 985-93.

[36] - Realistic distributions of infectious periods in epidemic models: changing patterns of persistence and dynamics., Theor Popul Biol, 60 (2001), 59-71.

[37] D. G. Luenberger, "Introduction to dynamic systems. Theory, models, and applications," John Wiley \& Sons Ltd., 1979.

[38] Z. M. Ma, J. Liu, and J. Li, Stability analysis for differential infectivity epidemic models, Nonlinear Anal. : Real world applications, (2003).

[39] C. Connell McCluskey, A model of HIV/AIDS with staged progression and amelioration., Math. Biosci., 181 (2003), 1-16.

[40] C. Connell McCluskey and P. van den Driessche, Global analysis of two tuberculosis models., J. Dyn. Differ. Equations, 16 (2004), 139-166.

[41] M. A. Nowak and R. M. May, "Virus dynamics. Mathematical principles of immunology and virology," Oxford University Press, 2000.

[42] C. P. Simon and J. A. Jacquez, Reproduction numbers and the stability of equilibria of SI models for heterogeneous populations, SIAM J. Appl. Math., 52 (1992), 541-576.

[43] Carl P. Simon, John A. Jacquez, and James S. Koopman, A Lyapunov function approach to computing R0, in "Models for infectious human diseases: their structure and relation to data," V. Isham and G. Medley, eds., Cambridge University Press, 1996, 311-314.

[44] Horst R. Thieme, Mathematics in population biology, Princeton Series in Theoretical and Computational Biology, Princeton University Press, Princeton, NJ, 2003.

[45] P. van den Driessche and J. Watmough, reproduction numbers and sub-threshold endemic equilibria for compartmental models of disease transmission, Math. Biosci., (2002).

Received September 2006; revised May 2007; final June 2007.

E-mail address: iggidr@math.univ-metz.fr

E-mail address: mbangjoh@yahoo.fr

E-mail address: sallet@loria.fr

E-mail address: tewajules@yahoo.fr 